\title{
Industrie 4.0? Mit Sicherheit!
}

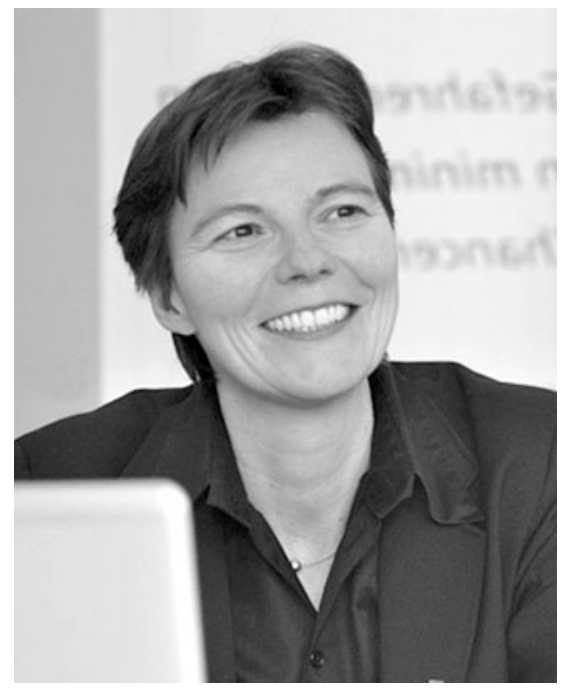

Digitalisierung und flächendeckende Vernetzung verändern rasant alle gesellschaftlichen und wirtschaftlichen Bereiche. Unter dem Schlagwort Industrie 4.0 werden die mit dieser Digitalisierung einhergehenden Veränderungen insbesondere bei den industriellen Produktions-, Fertigungs- und Automatisierungsprozessen zusammengefasst.

Industrie 4.0 bringt das Internet of Things (IoT), das Internet der Dienste und das Internet der Menschen zusammen. Durch das Internet der Dinge werden physische Objekte und IKT-Objekte miteinander vernetzt. Die physischen Objekte werden smart. Sie können als smarte Produkte, als smarte Werkstücke oder smarte Werkzeuge ihre eigene Produktion aktiv steuern, um auf diese Weise Produktionsprozesse effizient und qualitativ hochwertig zu gestalten. Die physischen Objekte erhalten eine virtuelle Repräsentation, die beispielsweise in einer Cloud für verschiedene Dienstleistungen im Sinne des Internet der Dienste zugreifbar gemacht werden kann.

In vernetzten Industrie 4.0-Systemen spielen Daten, wie beispielsweise Daten über den Herstellungsprozess, eine herausragende Rolle und stellen zu schützende Werte dar. So werden über eine Vielzahl von vernetzten Sensoren kontinuierlich Daten über den Produktionsprozess, den Wirkbetrieb oder die zugrunde liegenden Logistikprozesse erfasst und in Cloud-Plattformen abgelegt. Neue, Daten-getriebene Dienstleistungen entstehen, die mittels intelligenter Datenanalyse-Verfahren die Daten zu mehrwertigen Diensten kombinieren. Ein Beispiel ist die vorrausschauende Wartung (predictive maintenance), um Leerlauf oder Schäden an einer Anlage zu verhindern.

Die Produktion wird immer stärker individualisiert und personalisiert, was durch den Übergang zu einer Losgröße 1 charakterisiert wird. Dies erfordert software-intensive Systeme mit einer Art AppStore für smarte Fabriken, so dass flexible Anpassungen von Produktionsprozessen zur individuellen Fertigung schnell durchgeführt werden können. Da Industrie 4.0 auch das Internet der Dinge mit dem Internet der Menschen kombiniert, entstehen völlig neue Assistenzsysteme und Arbeitswelten. Das bedeutet, dass die Roboter ihre „Käfige" verlassen und in enger Kooperation zwischen Mensch und Robotern neue Formen der computergestützten Kollaboration entstehen. Durch die Vorort Produktion können die individualisierten Produkte schnell über optimierte Logistiknetze zu den Kunden gelangen. Damit kann auch in einem Hochlohnland wie Deutschland weiterhin wettbewerbsfähig produziert werden.

Den offensichtlichen Chancen, die die Entwicklung hin zur Industrie 4.0 für den Hightech-Standort Deutschland mit sich bringt, stehen jedoch ganz erhebliche Herausforderungen insbesondere in Bezug auf die Informationssicherheit, den Datenschutz und auch der Funktions- und Ausfallsicherheit gegenüber. Der Schutz der Anlagen, Komponenten und Produkte vor gezielten Manipulationen, die Gewährleistung einer durchgehend hohen Verfügbarkeit der Produktionsprozesse, aber auch der Know-How-Schutz, oder die Sicherstellung der Echtzeitfähigkeit der Abläufe sind Beispiele für die zu bewältigenden großen Herausforderungen. So könnten manipulierte Sensoren beispielsweise mit gefälschten oder veralteten Daten Abläufe stören, Maschinen schädigen oder sogar die Betriebssicherheit gefährden.

Das vorliegende Themenheft beleuchtet mit unterschiedlichen Schwerpunkten und aus unterschiedlichen Blickwinkeln wesentliche Fragestellungen zur Thematik Sicherheit in der Industrie 4.0. Die Beiträge von Holger Junker und Thomas Hemker geben einen Überblick über die Bedrohungslage und die damit verbundenen Herausforderungen. Konkrete Lösungsansätze, um Steuerungskomponenten vor Manipulationen zu schützen und einen unerwünschten Know-How-Abfluss zu verhindern, werden in den Beiträgen von Norbert Wiedermann, Anna Fischer / Michael Gröne / Christian Stüble sowie Christian und Christoph Thiel aufgezeigt. Die zentrale Problematik des Vertrauens wird in dem Beitrag von Arno Fiedler / Christoph Thiel diskutiert. Ruth Stock-Homburg / Young-kul Park / Christian Holthaus setzen sich grundlegend mit der Rolle des Datenschutzes im Zeitalter der flächendeckenden Datenanalysen auseinander, während Frederic Fredersdorf / Judith Schwarzer / Dominik Engel sich auf die Frage des Datenschutzes bei Smart Metern konzentrieren. In dem abschließenden Beitrag geben Silke Jandt / Tatami Michalek / Kai Dietrich einen Ausblick auf die neue EU-Verordnung eIDAS und diskutieren, welche Auswirkungen sich im Hinblick auf die Beweiskraft digitaler Dokumente daraus ableiten lassen.

Ich wünsche allen Leserinnen und Lesern eine anregende Lektüre.

\section{Claudia Eckert}

